\title{
The Role of Cymbopogon Citratus Extract in Protecting the Liver Against Injurious Effect of Dimethylnitrosamine in Rats
}

\author{
Naglaa Ali Saber Sarg', Usama Fouad Ahmed ${ }^{1}$, Ali Mohamed Ali, Saleh Sayed Idris ${ }^{2,}$, \\ ${ }^{1}$ Department of Anatomy, Faculty of Medicine, Benha University, Benha City, Egypt \\ ${ }^{2}$ Department of Health Education \& Health Promotion, Umm Al-Qura University, Makkah City, Saudi Arabia
}

Email address:

Salehsidris57@hotmail.com (S. S. Idris)

\section{To cite this article:}

Naglaa Ali Saber Sarg, Usama Fouad Ahmed, Ali Mohamed Ali, Saleh Sayed Idris. The Role of Cymbopogon Citratus Extract in Protecting the Liver Against Injurious Effect of Dimethylnitrosamine in Rats. International Journal of Clinical and Developmental Anatomy.

Vol. 1, No. 4, 2015, pp. 89-95. doi: 10.11648/j.ijcda.20150104.12

\begin{abstract}
Background: N-Nitrosodimethylamine (NDMA) is a potent hepatotoxin that induces fibrosis of the liver. Cymbopogon citratus, a plant that is widely cultivated to be eaten either fresh with food or dried in tea or soft drink has been reported to possess a number of medicinal and aromatic properties. Aim of the work: This work is aimed to study the protective effect of Cymbopogon citratus ethanolic leaf extract against hepatic injury and fibrosis induced by repeated intermittent administration of Dimethylnitrosamine (DMN) in rats. Materials and methods: A total of 30 rats divided into 3 groups were used. Group 1 served as control, Group 2 was given intraperitoneal injection with DMA at a concentration of $10 \mathrm{mg} / \mathrm{kg}$ body weight on three consecutive days of each week over a period of three weeks. Group 3 was given DMA at a concentration of $10 \mathrm{mg} / \mathrm{kg}$ body weight on three consecutive days of each week over a period of three weeks and $100 \mathrm{mg} / \mathrm{kg}$ body weight of Cymbopogon citratus ethanolic aqueous leaf extract 5 hours after administration of DMN. Results: It was found that DMN produces histopathological changes of the liver; including signs of severe hepatic injury. The cytoplasm of most hepatocytes appeared vacuolated with pyknotic and karyolytic nuclei, congested blood vessels and focal necrotic areas with marked increase of the collagen fibers deposition around the blood vessels. Ultrastructural changes showed Pyknotic nuclei, vacuolated cytoplasm and reduction or complete loss of cristae in the mitochondria. These were reversed by simultaneous treatment with Cymbopogon citratus. In conclusion: The results of this present study indicated that aqueous leaf extracts of Cymbopogon citratus has an antihepatotoxic action against DMN induced hepatic oxidative damage in rats which might be ascribed to its antioxidant and free radical scavenging property.
\end{abstract}

Keywords: Dimethylnitrosmine, Fibrosis, Liver, Rat, Cymbopogon Citratus

\section{Introduction}

The liver is the largest visceral organ in the human body and the chief site for metabolism and detoxification. Liver diseases often progress from sub-clinical icteric hepatitis to necro-inflammatory hepatitis, fibrosis, cirrhosis, and hepatocellular carcinoma [1]. Liver cirrhosis often results in high mortality and is also a risk factor in the development of hepatocellular carcinoma (HCC) [2], which rank the fifth of the cancer incidence worldwide [3].

Several experimental and clinical evidences have shown a common link between chronic liver injury, oxidative stress, activation of hepatic stellate cells (HSCs) and their transformation to myofibroblast- like cells, associated with increased production of extracellular matrix proteins during hepatic fibrosis [4].

Dimethylnitrosamine (DMN), is an industrial by-product or waste product of several industrial processes. Of more general concern, water treatment via chlorination or chloramination of organic nitrogen-containing waste water can lead to the production of DMA at potentially harmful levels, [5]. DMA's contamination of drinking water is of particular concern due to the minute concentrations at which it is harmful, the difficulty in detecting it at these concentrations, and to the difficulty in removing it from drinking water, [6]. DMA may be formed during cooking foods, especially cured meats and fish, that contain sodium nitrite as a preservative, but is also found in several 
vegetables, cheeses, alcoholic beverages and fruits, as a contaminant in rubber products and tobacco smoke, [7].

$\mathrm{DMN}$ is a potent hepatotoxin, carcinogen and mutagen which has been demonstrated to induce bridging fibrosis, necrosis and collapse of parenchymal framework of liver, [8]. Hepatic cells are involved in a variety of metabolic events; therefore the establishment of liver protective/therapeutic agents is of a paramount importance in the protection of the liver from damage. Natural remedies from traditional plants are seen as effective and safe alternative treatments for hepatotoxicity, [9]. Cymbopogon citratus, commonly known as lemon grass, is a tropical monocotyledonous hypogeal perennial herb belonging to the family Poaceae. is widely cultivated to be eaten either fresh with food or dried in tea or soft drink has been reported to possess a number of medicinal and aromatic properties, its oil is use of more general levels as culinary flavoring, scent, and medicine, [10].

The present investigation was undertaken with the objective of evaluating the anti-fibrotic, antioxidant and protective efficacy of ethanolic leaf extract of Cymbopogon citratus against repeated DMN-induced hepatic fibrosis in rats.

\section{Materials and Method}

\subsection{Animals}

Thirty adult male albino rats were used in this study. Their weight ranged from 200-250 gm. Before commencing experimentation, all the animals were subjected to one week period of passive preliminaries in order to adapt themselves to their new environment at animal bread house in Anatomy department in Benha faculty of medicine. They were housed in clean well ventilated cages under strict care and hygiene. The rats were provided with standard laboratory diet and water.

\subsection{Chemicals}

a N-Nitrosodimethylamine:

NDMA obtained from, faculty of science, Biochemistry department, Benha university. 10mg of DMA dissolved in 10 $\mathrm{ml}$ of sodium hydrochloride, so every $1 \mathrm{ml}$ solution contains 1 mg of the drug.

b C-citratus leaves Extraction:

The collected $\mathrm{C}$. citratus leave was cleaned and oven-dried at $65^{\circ} \mathrm{C}$ and coarsely ground. The powdered materials of $\mathrm{C}$. citratus $(100 \mathrm{~g})$ were extracted with $1000 \mathrm{~mL}$ of $70 \%$ methanol for 3 days with occasional shake. The plant was filtered through filter paper and this procedure was repeated three times. The extracts were then evaporated to eliminate the methanol. The extracts were then stored at $-20^{\circ} \mathrm{C}$ until used. The yields were $12.34 \mathrm{~g}$. The powdered extracts were reconstituted with distilled water to the desired concentrations prior to use.

\subsection{Experimental Design}

The animals were divided into three groups of ten rats each:
The liver of the three groups were extracted. The specimens of liver were divided into two parts. One part was fixed in $10 \%$ formaldehyde for light microscopic study, while the other part was fixed in $2 \%$ cacodylate buffered gluteraldehyde solution for $2-4$ hours at $4^{\circ} \mathrm{C}$ for electron microscopic study.

For light microscopy, the slides were stained by:

(1) Hematoxylin and Eosin stain [11].

(2) Periodic Acid Schiff's stain (PAS) [12].

(3) Masson's trichrome stain [13].

For electron microscopic examination, the ultrathin sections were stained by uranyl acetate and lead citrate and examined with Joel CX 100 transmission electron microscope in laboratory of the histology and cell Biology Department, Faculty of Medicine Benha University.

\section{Results}

Light microscopic examination of liver sections of an adult control rat normal hepatic architecture where the hepatocytes were arranged in cords radiating from the central vein extending to the periphery of the lobule. The cords were separated from each other by blood sinusoids which lined by flat endothelial cells and few kuppfer cells. The hepatocytes showed an acidophilic cytoplasm and rounded vesicular nuclei with one or two prominent nucleoli [Fig. 1]. The PAS preparations of the normal liver of rats revealed that the glycogen granules were observed in the cytoplasm of the hepatocytes as indicated by large number of magenta red fine granules [Fig. 2]. In Masson's trichrome stain, the connective tissue around the portal tract was scanty [Fig. 3].

The ultrastructural picture of the hepatic tissue in the same group revealed, normal hepatic cell with rounded nucleus and prominent nucleolus, tight junction and bile canaliculi between adjacent hepatocytes [Fig. 4]. The cytoplasm was crowded with cell organelles, the most numerous were mitochondria with regularly arranged cristae in close association appeared variable in sizes and shapes, glycogen granules and the rough endoplasmic reticulum were located near the nucleus [Fig. 5].

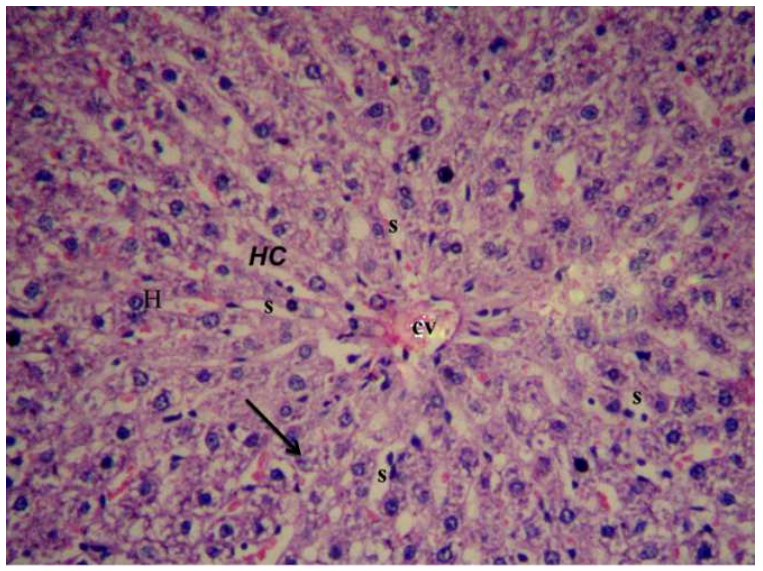

Fig. 1. A photomicrograph of liver section of control rat showing: Cords $(H C)$ of hepatocytes $(H)$ radiating from the central vein $(C V)$. Notice Kupffer cells (black arrow) bulge in blood sinusoids (S). [Hx \&E X200]. 


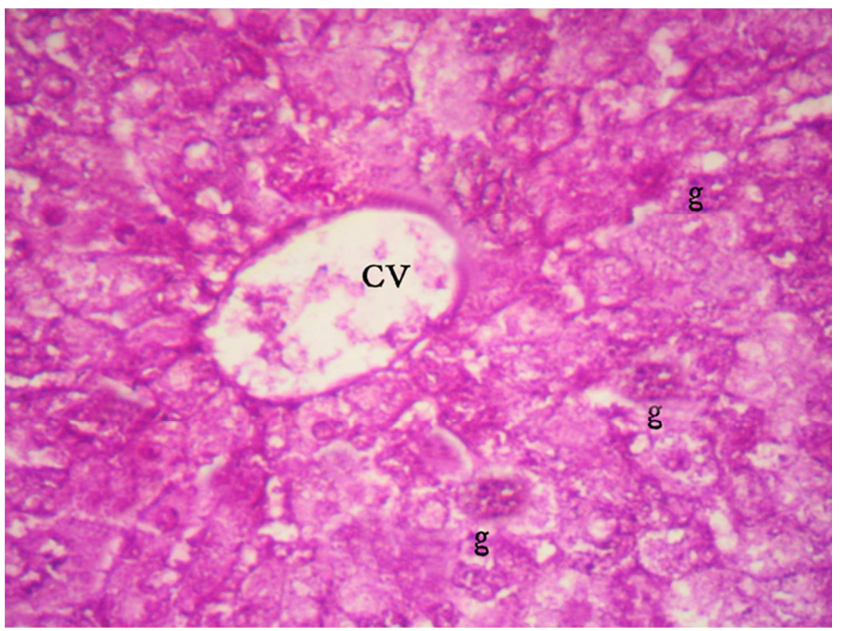

Fig. 2. A photomicrograph of liver section of control rat showing: Positive $P A S$ reaction for glycogen granules (g) in the cytoplasm of hepatocytes. Notice the central vein (CV). [PAS X 200].

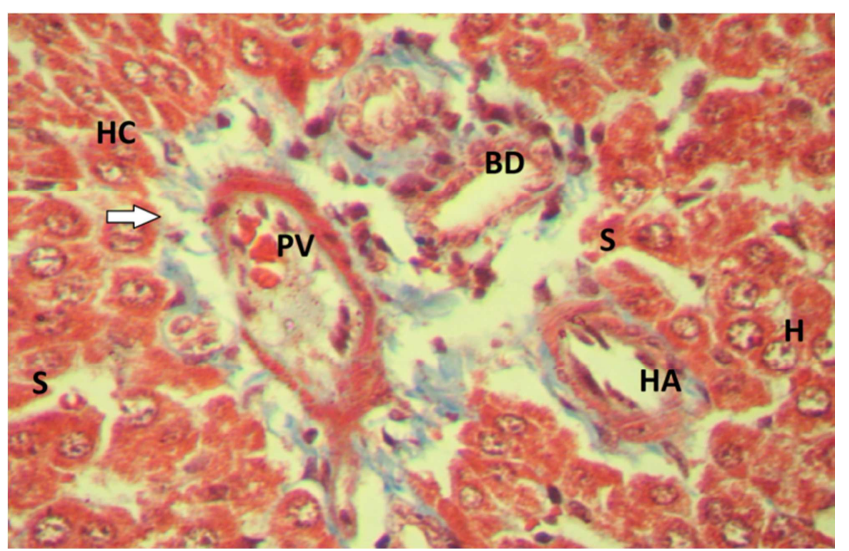

Fig. 3. A photomicrograph of liver section of control rat showing: Scanty perivascular connective tissue (arrow) around portal tract containing a branch of hepatic artery (HA), a branch of portal vein (PV) and bile ducts (BD). Notice cords of hepatocytes (H) and blood sinusoids (S) between hepatic cords (HC). [Masson's Trichrome X400].

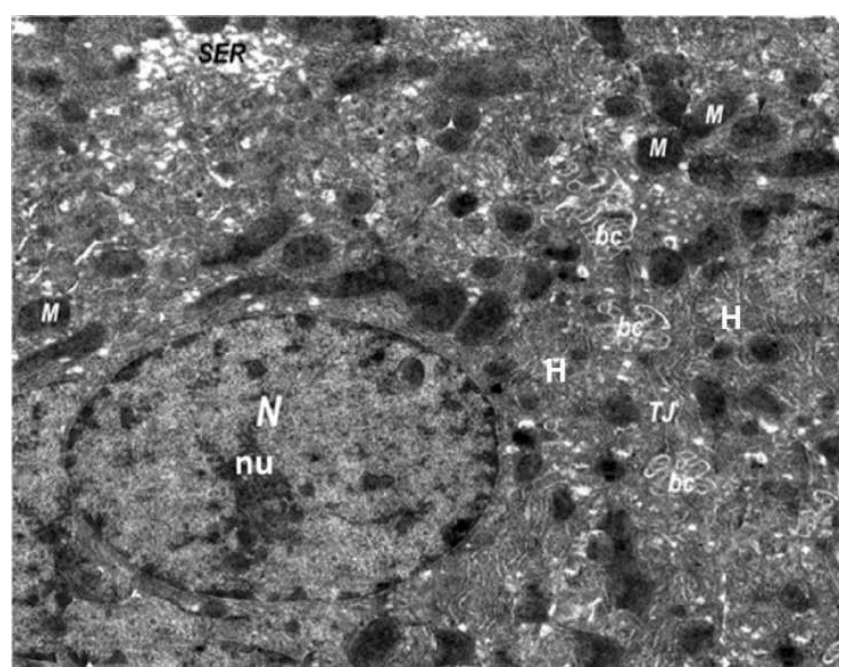

Fig. 4. An electron photomicrograph of ultrathin section in liver of control rat showing: The hepatocyte $(H)$ with rounded nucleus $(N)$ and nucleolus (nu). The cytoplasm contains many mitochondria $(M)$, smooth endoplasmic reticulum (SER). Notice the canaliculi (bc) and tight junction (TJ) in between the cells. [E. MX 6000].

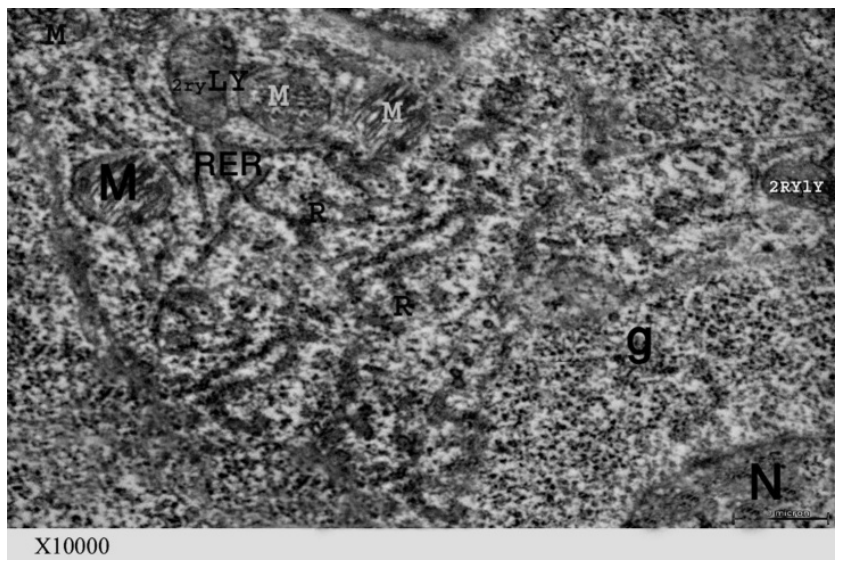

Fig. 5. An electron photomicrograph of ultrathin section in liver of control rat showing: The cytoplasm contains mitochondria (M), glycogen granules (g), rough endoplasmic reticulum (RER) and lysosomes (LY). Notice a part of nucleus (N) of hepatocyte. [E.MX 10000].

The light microscopic examination of rats which were exposed to nitrosodimethylamine for 3 weeks showed clear signs of severe hepatic injury manifested by lose of normal hepatic architecture, dilated and congested central vein. The cytoplasm of most hepatocytes appeared vacuolated area with pyknotic or, karyolytic nuclei (Fig. 6). Most hepatocytes appeared vacuolated [Fig. 7]. After exposure of rats to DMN, the glycogen granules of the liver cells obviously were decreased [Fig. 8]. After Masson's trichrome staining there was marked increase of the collagen fibers deposition around the portal tirades compared with the control one [Fig. 9].

The ultrastructural picture of the hepatic tissue in the same group revealed that some cells showed the nuclei frequently appeared with irregular shapes and chromatin condensation. Other damaged cells appeared with pyknotic nuclei, vacuolated cytoplasm [Fig. 10] and little or no glycogen granules. The mitochondria showed a reduction or complete loss of cristae and an amorphous or granular matrix [Fig. 11].

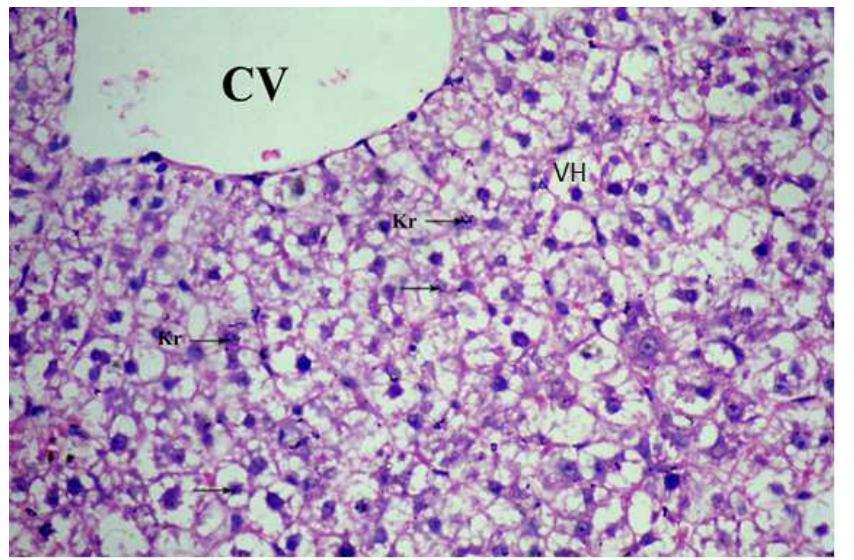

Fig. 6. A photomicrograph of liver section of rat treated with DMN showing: dilated central vein $(C V)$, loss of normal hepatic architecture. The cytoplasm of hepatocytes is vacuolated (VH). Notice the kupffer cell (kr). [Hx \&E $X 400]$. 


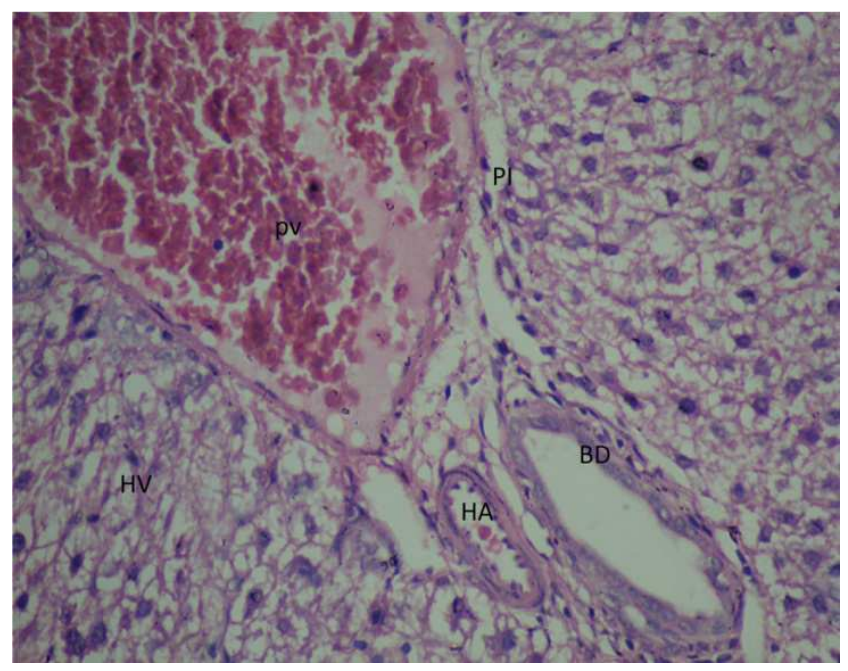

Fig. 7. A photomicrograph of liver section of rat treated with DMN showing: Portal tract containing a branch of hepatic artery (HA), congested and dilated portal vein $(P V)$ and bile duct $(B D)$., Notice hepatocytes are vacuolated (HV). [Hx\& E X400].

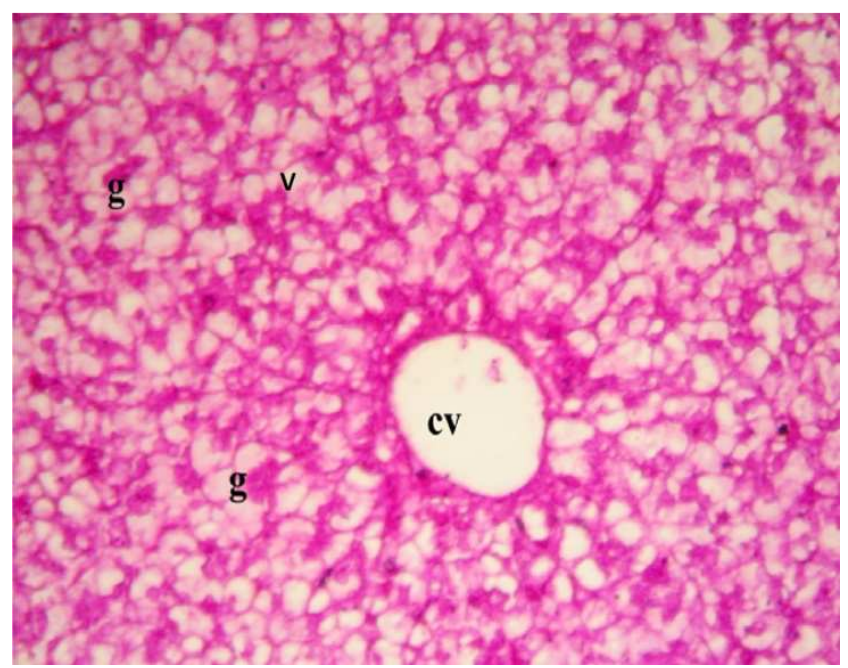

Fig. 8. A photomicrograph of liver section of rat treated with DMN showing. Vacuolations in liver cells (V) with depletion of glycogen granules (g). Notice the central vein. [PAS X 200].

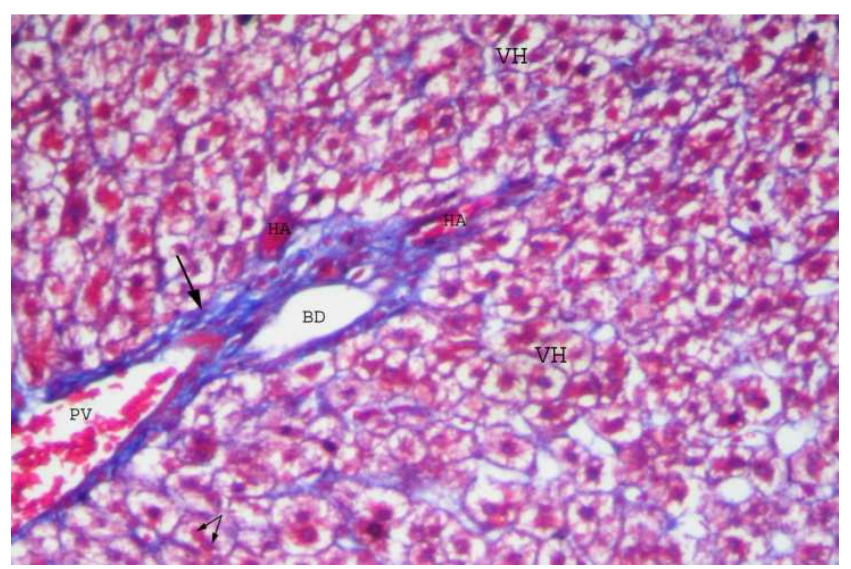

Fig. 9. A photomicrograph of liver section of rat treated with DMN showing: Increase in connective tissue (arrow) around the portal tract. Notice the congested portal vein $(P V)$, hepatic artery (HA) and bile duct. Notice the vacuolated hepatocytes (VH). [Masson's Trichrome X200].

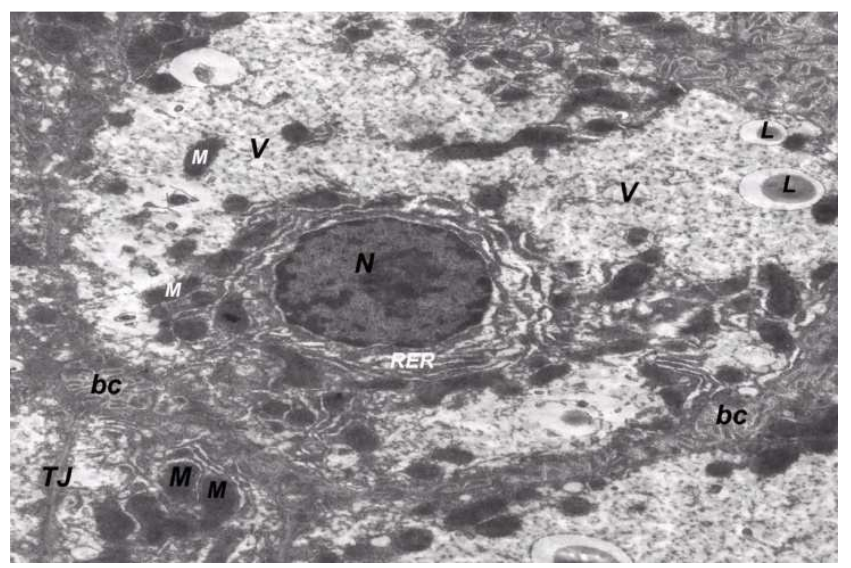

Fig. 10. An electron photomicrograph of ultrathin section in liver of rat treated with DMN shows the nucleus (N) with irregular shape and chromatin condensation. The cytoplasm shows large vacuoles, mitochondria (M), condensed rough endoplasmic reticulum (RER) around the nucleus and lysosomes (L). Notice the tight junction (TJ) and bile canaliculi (bc). (E. MX 6000).

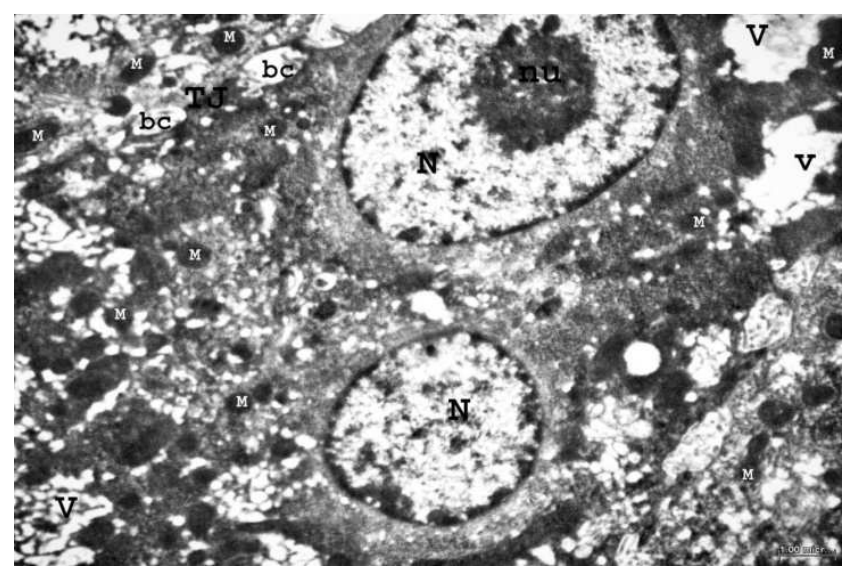

Fig. 11. An electron photomicrograph of ultrathin section in liver of rat treated with DMN showing: The nuclei of hepatocytes $(N)$ are vacuolated (V) with collection of chromatin materials in the center of nucleus. The cytoplasm contains vacuoles (V) of some degenerated mitochondria (M). Notice the bile canaliculi (bc). [E. MX 6000].

The light microscopic examination of rats which were exposed to nitrosodimethylamine and C. citratus aqueous extract showed signs of improvement through a well arrangement of hepatocytes with normal cytoplasm. Sinusoids were well preserved and some hepatocytes showed vacuolated cytoplasm compared with rats treated with nitrosodimethylamine only [Fig. 12]. In PAS staining, most of hepatocytes had good positive PAS for glycogen granules and few hepatocytes had poor PAS reaction [Fig. 13]. In Masson's trichrome stain, the connective tissue around the central vein and portal tract was decreased when compared with the nitrosodimethylamine group [Fig. 14].

The ultrastructural picture of the hepatic tissue in the same group showed some improvement, the hepatocytes appear normal with rounded nucleus and nucleolus. The cytoplasm contains numerous organelles. Some mitochondria are numerous and intact, with apparent cristae. Rough endoplasmic reticulum was preserved. Lipid droplets, glycogen granules, multivesicular bodies, and few vacuoles 
were also appeared [Fig. 15]. The bile canaliculi were found in between the adjacent hepatocytes, containing microvilli of the hepatocytes [Fig. 16].

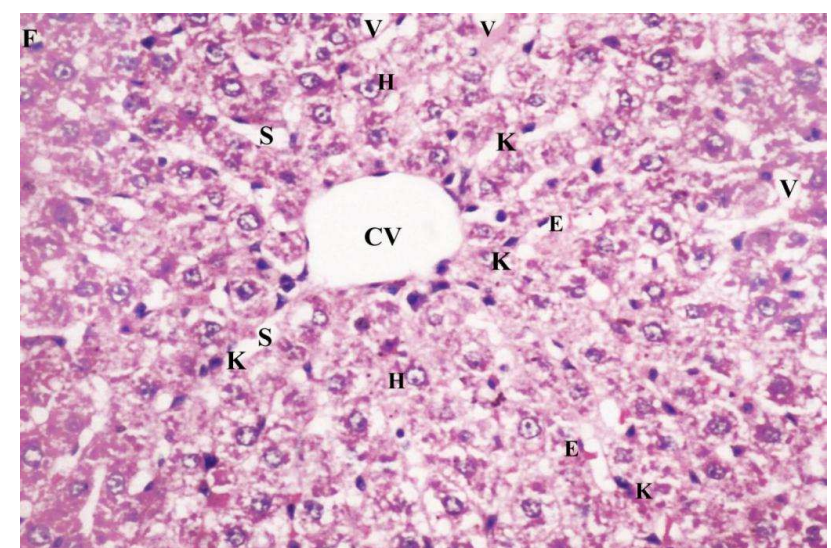

Fig. 12. A photomicrograph of liver section of rat treated with DMN and $C-$ Citratus showing: Cord of hepatocytes $(H)$, blood sinusoids $(S)$ around the central vein $(\mathrm{CV})$ which is lined by endothelial cells. Some hepatocytes $(H)$ are intact while the others are vacuolated $(V)$. Noticed the Kupffer cells $(K)$ bulge in blood sinusoids. [Hx \& E X200].

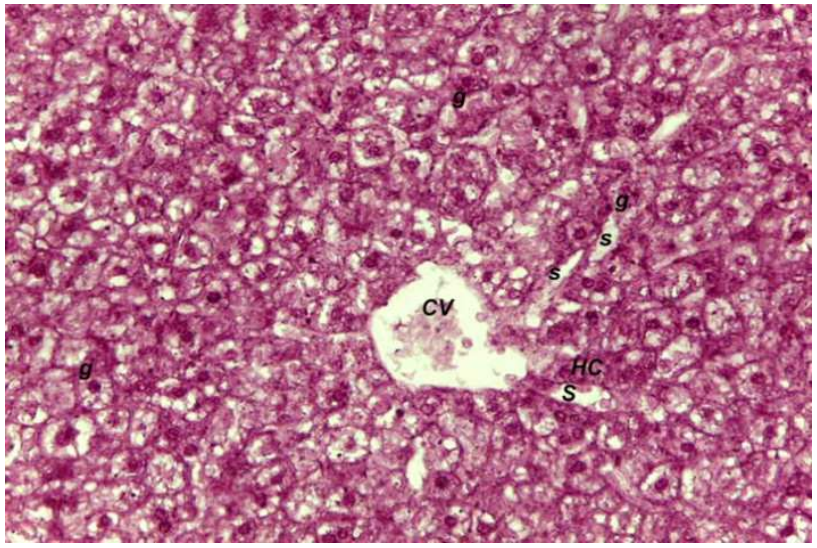

Fig. 13. A photomicrograph of liver section of rat treated with DMN and $C$ citratus showing: Positive PAS reaction for glycogen granules $(g)$ in the cytoplasm of hepatocytes. Notice central vein (CV) and hepatic cords $(\mathrm{HC})$ separated by blood sinusoids (S). [PAS X 200].

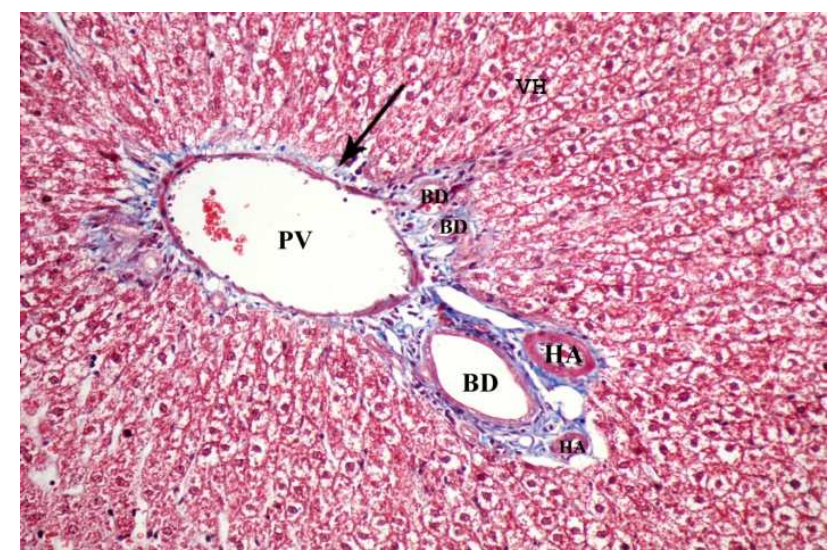

Fig. 14. A photomicrograph of liver section of rat treated with DMN and Ccitratus showing, decreased perivascular connective tissue (arrow) around portal tract. It contains dilated portal vein $(P V)$, hepatic artery $(H A)$ and bile ducts (BD). Some hepatocytes are still vacuolated (VH). Notice preserved hepatic architecture. [Masson's Trichrome X200].

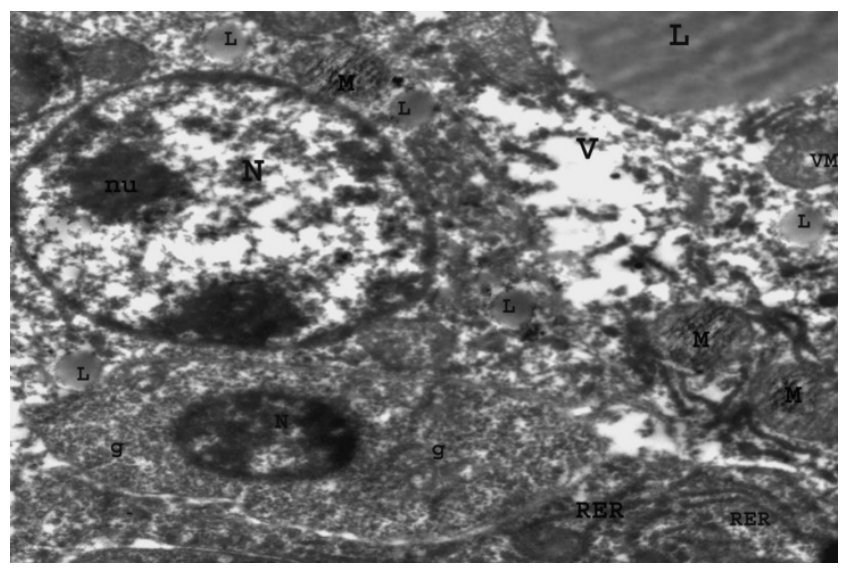

Fig. 15. An electron photomicrograph of ultrathin section in liver of rat treated with DMN and C-citratus showing: Hepatocytes with oval nucleus (N) The cytoplasm contains numerous mitochondria $(M)$, lipid droplet $(L)$, rough endoplasmic reticulum (RER), and few vacuolations (V). [E. MX $6000]$.

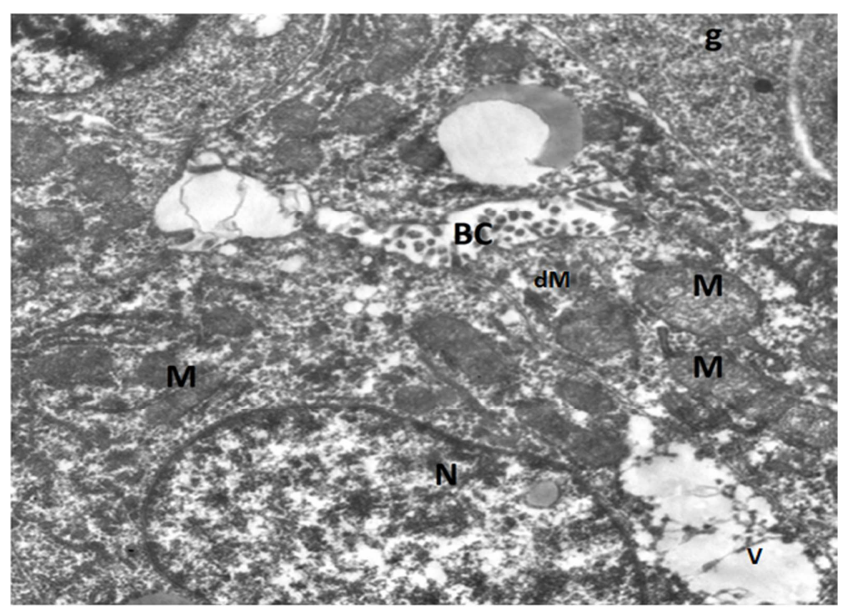

Fig. 16. An electron photomicrograph of ultrathin section in liver of rat treated with DMN and C-citratus showing: The hepatocyte has large oval nucleus (N) with dispersed chromatin materials. The cytoplasm contains some intact mitochondria (M), other are degenerated (dM) and small sized vacuoles $(V)$. Notice the bile canaliculi (bc) in between the hepatocytes. [E. $M X 10000]$

\section{Discussion}

It is well known that liver toxicity is one of the most important problems in the world. Therefore, the efforts in the field of hepatoprotection and treatment of liver disorders are very much required. NDMA is highly toxic to experimental animals. A large single or multiple small doses produce severe hepatic injuries, tumors or leukemias [14]. Fibrosis as a scarring response to liver damage constitutes the injurious process that can ultimately lead to impairment of liver function, development of hepatocellular carcinoma, and portal hypertension with all its associated complications [15].

In the present study, the light microscopic study revealed many histological changes in the liver of rats that received DMN. These changes were in the form of diffuse vacuolations in the cytoplasm of the hepatocytes and increased connective tissue around the dilated and congested blood vessels and hepatic sinusoids. This was in agreement 
with [16]; who found that animals treated with NDMA exhibited perivenular necrosis and micro vesicular fatty change in peripheral hepatocytes. There were severe centrilobular congestion and marked dilatation of central vein and sinusoids with massive necrosis and initiation of fibrosis. Also, these results were similar to the study of [17] that showed, in the Hx \& E stained rat liver cells of DMN treated rats, a high degree of centrilobular necrosis and bridging fibrosis between the portal triad and the central vein.

In the current work, PAS-stained sections of the control group showed normal content of glycogen granules evidenced by a strong PAS reaction (in the form of mahogany coloration). Also, these granules were evident ultrastructurally in the control group. In contrast, the DMNtreated group showed a weak PAS reaction indicating depletion of glycogen. The reduction in carbohydrate contents could be attributed to increased stress on the liver, leading to the consumption of high energy in an attempt to reduce or equalize the pressure exerted on it [18].

In the current study the ultrastructural changes showed electron-lucent areas of the cytoplasm in hepatocytes in rats that received DMN. Other degenerative changes were also observed. These changes include lipid droplets deposition in the cytoplasm of hepatocytes, decreased organelles, damaged cristae and vacuoles in mitochondria, dilated endoplasmic reticulum and decrease of glycogen granules. In this research, there were variations in the size and shape of nuclei of the liver cells. Most of hepatocytes had extended chromatin with marginal displacement of their nucleoli and peripheral condensation of their nuclear chromatin which might be attributed to cellular hyperactivity. Some of the hepatocytes had shrunken electron-dense nuclei. The variations of the shape of nuclei were explained by [19], to be due to compression of nuclei by vacuoles in the cytoplasm of hepatocytes. However, the variations in size of nuclei may be due to the increase in liver DNA content. The endoplasmic reticulum was particularly liable to the free radical attack, not only because it is considered as a site of radical production but also because its membrane is rich in polyunsaturated fatty acids which are susceptible to free radical attack. Therefore, the dramatic ultrastructural changes observed in the present study may be attributed to increased oxidative stress as reported by [20].

The mitochondrion was the major target in drug-induced hepatic damage [21]. Mitochondrial dysfunction was generally accompanied with oxidative stress, which is a key regulator of mitochondria-mediated cell death. Hence, drugs cause cell death by inducing oxidative stress in hepatic mitochondria. [22]. The cytoplasmic vacuolations could be attributed to mitochondrial degeneration. The mitochondrial damage probably occurs because of the electrolyte imbalance or edema because of the inhibition of the membrane enzyme of the sodium pump [23].

The increase in the lipid peroxidation of mitochondria could lead to inhibition of respiratory enzymes which in turn could inhibit ATP production. The decrease in intracellular ATP leads to mitochondrial damage [24].
In the present investigation, amelioration of $\mathrm{DMN}$-induced hepatic fibrosis, inflammatory changes and hepatic necrosis was well evidenced in rats treated with $\mathrm{C}$. citratus aqueous extract with DMN simultaneously. Light microscopic examination of the liver sections showed a more or less similar picture to those of the control group with relative restoration of the general hepatic lobular architecture. The central vein was surrounded by cords of relatively normally appearing hepatocytes and blood sinusoids. Some hepatocytes were binucleated and others still had a vacuolated cytoplasm and small dark nuclei. However, still there was dilated central vein. A strong PAS reaction could be observed in this group. And this was in line with the results of [25], who found an improvement of liver tissue in rats treated with. C. citratus aqueous extract after DMN intoxication. [10] Found that the liver tissue of rats treated with $\mathrm{C}$. citratus aqueous extract with oxidative stress induced by $\mathrm{H} 2 \mathrm{O} 2$, exhibited a similar liver cytoarchitecture compared with the control group. The hepatoprotective effect of $\mathrm{C}$. citratus might be ascribable to its antioxidant and free radical scavenging property [26]. [27], reported that the leaves of $C$. citratus contained saponins, sesquiterpenes, lactones, steroids, flavonoids. Flavonoids were reported to exhibit antioxidant activity ([28] and were effective scavengers of superoxide anions [29]. The aqueous extract of $C$. citratus may have exhibited hepatoprotective activity due to its possible antioxidant content attributable to flavonoids. Interestingly, saponins especially terpene glycosides were reported to enhance natural resistance and recurative powers of the body [30].

\section{Conclusion}

The use of aqueous leaf extracts of Cymbopogon citratus was found to have a protective action against DMN induced hepatic oxidative damage in rats. This was due to its antioxidant and free radical scavenging property.

\section{References}

[1] Lima CF, Fernandes-Ferreira M and Pereira-Wilson C (2007): Drinking of Salvia officinalis tea increases CCl4-induced hepatotoxicity in mice. Food Chem Toxicol 45 (3): 456-464.

[2] Bataller R and Brenner DA (2005): Liver fibrosis. J Clin Invest 115 (2): 209-218.

[3] Caldwell S and Park SH (2009): The epidemiology of hepatocellular cancer: from the perspectives of public health problem to tumor biology. J Gastroenterol 44 (suppl. 19): 96-101.

[4] Vendemiale G, Grattagliano I, Caruso ML, Serviddio G and Valentini AM (2001): Increased oxidative stress in dimethylnitrosamine-induced liver fibrosis in the rat: effect of $\mathrm{N}$-acetylcysteine and interferon-alpha. Toxicol Appl Pharm acol 175 (2): 130-139.

[5] David L. Sedlak, Rula A. Deeb, Elisabeth L. Hawley, William A. Mitch, Timothy D. Durbin, Sam Mowbray and Steve Carr(2005): Sources and Fate of Nitrosodimethylamine and Its Precursors in Municipal Wastewater Treatment Plants Water Environment Research Vol. 77, No., pp. 32-39. 
[6] Mitch, W. A.; Sharp, J. O.; Trussell, R. R.; Valentine, R. L.; Alvarez-Cohen, Land Sedlak, D. L. (2003): NNitrosodimethylamine (NDMA) as a Drinking Water Contaminant. Environmental Engineering Science 20 (5): 389-404.

[7] Najm, I.; Trussell, R. R. (2001): NDMA Formation in Water and Wastewater. Journal American Water Works Association 93 (2): 92-99.

[8] George J, Rao KR, Stern R and Chandrakasan G (2001): Dimethylnitrosamine induced liver injury in rats: the early deposition of collagen. Toxicology 156 (2-3): 129-138.

[9] Choi JH, Kim DW, Yun N, Choi JS and Islam MN (2011): Protective effects of hyperoside against carbon tetrachlorideinduced liver damage in mice. J Nat Prod 74: 1055-1060.

[10] Saleh Muhammad Rahim, Ekhlass Muhi Taha2, Muneef Saeb Al-janabi1, Bushra Ismael Al-douri1, Kumar Das Simon3, Abd Gaffar Mazlan3 (2014): Hepatoprotective Effect Of Cymbopogon Citratus Aqueous Extract Against Hydrogen Peroxide-Induced Liver Injury In Male Rats. Afr J Tradit Complement Altern Med. 11(2): 447-451.

[11] Bancroft J. D. and Gamble M. (2008): Theory and practice of histological techniques. 6th ed. Churchill Livingstone. London, New York \& Sydney, P. 121-132.

[12] Horbin E. and Bancroft, J. D. (1998): Trouble shooting histology stain, PAS technique. Churchill, Livingstone, New York. P. 154.

[13] Leong, A. S. (1996): Principles and practice of medical laboratory science. Volume 1: Basic Histotechnology. 1st ed., Philadelphia, Saunders Company. P. 171.

[14] Ahmad R. (2009): Operculina turpethum attenuates Nnitrosodimethylamine induced toxic liver injury and clastogenicity in rats. Chem Biol Int.; 181: 145-153.

[15] Usunomena U, Okolie N. Paulinus and Eze I. Gerald (2015): Inhibitory Effect of Vernonia amygdalina on Dimethylnitrosamine (DMN)-induced Liver Fibrosis in Rats. Int J Clin Pharmacol Toxicol, 4(4) 179-184.

[16] Sharma, V and Singh M. (2014): Attenuation of nitrosodimethylamine induced hepatotoxicity by Operculina turpethum in Swiss Albino mice. Iran J Basic Med Sci. Jan; 17(1): 73-80.

[17] Ezhilarasan, D. Karthikeyan, S. and Palani Vivekanandan (2012): Ameliorative effect of silibinin against Nnitrosodimethylamine-induced hepatic fibrosis in rats. Environmental toxicology and pharmacology. (34)1004-1013.

[18] Sakr S. A., Abdel Samei H. A. and Soliman M. E. (2004): Exploring hepatotoxicity of benomyl. Histological and histochemical study on albino rats J Med Sci; 4: 77-83.
[19] El-Roby N. M. (1994): Histological and Histochemical Effects of Some Steroid Hormones on the Liver of Male Rats. Thesis of M. D. Cairo University. 56-88.

[20] Pradeep, K.; Raj Mohan, C. V.; Gobianand, K and Karthikeyan, S. (2010): Protective effect of Cassia fistula Linn. on diethylnitrosamine induced hepatocellular damage and oxidative stress in ethanol pretreated rats. Biol. Res., 43(1): 113-25.

[21] Kass, G. E. (2006): Mitochondrial involvement in druginduced hepatic injury. Chem - Biol. Interact., 163: 145-159.

[22] Boelsterli, U. A., and Lim, P. L. (2007): Mitochondrial abnormalities - a link to idiosyncratic drug hepatotoxicity. Toxicol. Appl. Pharmacol., 220: 92-107.

[23] Gowtham Kumar Subbaraj, Langeswaran Kulanthaivel, Revathy Rajendran Ramakrishnan Veeraba Thiran (2013): Ethanolic Extract of Carum Carvi (Eecc) Prevents NNitrosodiethylamine Induced Phenobarbital Promoted Hepatocarcinogenesis By Modulating Antioxidant Enzymes. International Journal of Pharmacy And Pharmaceutical Sciences Issn-0975-1491 Vol 5, Suppl 1.

[24] Pessayre D1, Mansouri A, Berson A and Fromenty B. (2010): Mitochondrial involvement in drug-induced liver injury. Handb Exp Pharmacol; (196): 311-65.

[25] Pei HK, Mokhtar RA and Mohammad I (2012): Antioxidant potential of Cymbopogon citratus extract: alleviation of carbontetrachloride-induced hepatic oxidative stress and toxicity. Hum. Exp. Toxicol. 311: 81-91.

[26] Koh, PH1, Mokhtar RA, Iqbal M. (2012): Antioxidant potential of Cymbopogon citratus extract: alleviation of carbon tetrachloride-induced hepatic oxidative stress and toxicity. Hum Exp Toxicol Jan; 31(1): 81-91.

[27] Omotade IO (2009): Chemical profile and antimicrobial activity of Cymbopogon citratus leaves. Journal of Natural Products, 2: 98-103.

[28] Ramanathan R, Luu K and Das NP (1989): Anti peroxidative action of flavonoids and related products in ground pork Abstracts proceedings of III International Symposium on flavonoids in Biology and Medicine. Singapore. p. 56.

[29] Robak k and Grygleuski RJ (1988): Flavonoids are scavengers of superoxide anions. Biochem. Pharmacol. 37: 837-841.

[30] Singh, N., Verma, P., Misharu, N. and Nath R (1991): A comparative evaluation of some antistress agents of plant origin. Indian J. Pharmacol. 21: 99. 